\title{
A review on novel magnetic properties of metamaterials CRLH devices : Part 1
}

\author{
Review \\ Article \\ Mahmoud A. Abdalla ${ }^{1}$ and Zhirun $\mathrm{Hu}^{2}$ \\ ${ }^{1}$ Military Technical College, Cairo, Egypt, ${ }^{2}$ University of Manchester, United \\ Kingdom
}

\section{Keywords:}

Couplers, CRLH TL, ferrite, impedance transformers.

\section{Corresponding Author:}

Zhirun $\mathrm{Hu}$, University of Manchester, United Kingdom.

Email: z.hu@manchester.ac.uk

\section{Abstract}

This paper introduces different varieties of novel composite right / left handed metamaterials devices with novel magnetic operation properties. The reported devices are discussed in terms of their theoretical. The reported devices have the advantages of their small size, multi frequency band in addition to broadband operation $(\approx 60 \%)$ compared to their conventional design. Moreover, these devices are designed to illustrate novel magnetic properties such as their tuning and non reciprocity operation capabilities. Theoretical explanations supported by simulation results of presented devices confirmed the operation of a tunable and nonreciprocal coupler and tunable transformer. The novelty and advantage of the reported devices are their combined properties of compactness and nonreciprocity. Furthermore the devices require very low external DC magnetic bias due to its much lower demagnetization compared to the microstrip configuration. Accordingly, the devices can be applied in many RF front end novel applications such as circulators and isolators.

\section{INTRODUCTION}

Electromagnetic Metamaterials (MTMs) were first introduced in 1967 by the Russian physicist Victor Veselago. He named MTMs that illustrate simultaneous negative electric permittivity $\varepsilon$ and permeability $\mu$ as lefthanded metamaterials (LHM) ${ }^{[1]}$. Thanks to theses negative parameter values, these LH MTMs, are characterized by unconventional electromagnetic propagation parameters. Not until of early of this century where LH MTMs were first experimentally demonstrated by pioneering works of Pendry by using array of split ring resonators (SRRs) with a wire array placed in space ${ }^{[2]}$. However, such model was not suitable for microwave applications. Therefore, planar LH MTM versions have been proposed for $\mathrm{RF} /$ microwave applications in both microstrip and coplanar waveguide $(\mathrm{CPW})$ configurations ${ }^{[3,4]}$. Due to effect of parasitic elements of hosting transmission line, these LH MTM is a composite right/left handed transmission line (CRLH TL). However, all these examples were suggested using dielectric substrates. Later on, it has been suggested using of ferrite substrates in implementing the CRLH TLs. Unlike dielectric materials, ferrite medium is nonreciprocal and has a dispersive permeability tensor where the permeability element value can be negative or positive depending on the applied DC magnetic bias. Thus a novel tunable and nonreciprocal CRLH transmission line can be realized. Examples of these new LH TLs have been introduced using microstrip configuration, and
(CPW) configuration ${ }^{[5,6]}$. CRLH CPW TL requires smaller DC magnetic bias than microstrip one due to its smaller demagnetization field.

Based on these transmission lines, the need for tunable and nonreciprocal microwave devices and antennas has encouraged researchers to realized tunable and nonreciprocal CRLH TLs using ferrite substrates ${ }^{[7-11]}$. Ferrite medium has nonreciprocal dispersive properties due to its dispersive permeability tensor ${ }^{[12]}$. The tuning characteristics are achieved by varying the applied DC magnetic bias. Over the ferrite CRLH TLs, it has proven that coplanar waveguide based devices needs smaller DC magnetic bias compared to microstrip configuration ${ }^{[7]}$. Examples for these devices include tunable/nonreciprocal couplers, resonators, circulators, phase shifter ${ }^{[13-26]}$ and antennas ${ }^{[27,28]}$.

In this paper, we introduce a survey on novel set of microwave devices implemented using ferrite substrate in CRLH MTMs configurations. These devices were implemented in CPW configuration to have the advantage of its small DC magnetic bias. Thanks to the employed CRLH configuration, the reported devices demonstrate the advantages of the compact size and the power enhancement. Moreover, these devices can be tunable and nonreciprocal thanks to the magnetic properties of the ferrite. The performance of these devices are explained theoretically and confirmed by both numerical simulations and measurements. 


\section{THEORY}

The ferrite CRLH TL can be realizable by loading a conventional hosting ferrite TL by a series capacitive load and shunt inductive load, similar to conventional (dielectric) CRLH TLs. The equivalent circuit of a lossless unit cell of ferrite CRLH TL of length (d), it is much smaller than the travelling wavelength, can be expressed as a standard lossless composite right/left handed CRLH unit cell shown in Figure 1. In the equivalent circuit, the parasitic inductance $\left(\mathrm{L}_{\mathrm{R}}\right)$ and capacitance $\left(\mathrm{C}_{\mathrm{R}}\right)$ of the hosting ferrite TL, while $\left(\mathrm{L}_{\mathrm{L}}\right)$ and $\left(\mathrm{C}_{\mathrm{L}}\right)$ are the shunt inductive and series capacitive load, respectively. The dispersion propagation constant $\left(\beta_{\mathrm{LH}}\right)$ for all reported CRLH TLs can be calculated approximately by expressing the hosting ferrite TL parameters in terms of its medium parameters, the ferrite medium permittivity and the equivalent relative ferrite permeability which depends of the DC magnetic bias direction as ${ }^{[5,6,29]}$.

$$
\cos \left(\beta_{L H} d\right)=1-\frac{1}{2} \omega^{2} d^{2}\left(\mu_{o} \mu_{f}-\frac{1}{\omega^{2} C_{L} d}\right)\left(\varepsilon_{o} \varepsilon_{f}-\frac{1}{\omega^{2} L_{L} d}\right)
$$

In case of ferrite CRLH CPW TL, the DC magnetic bias direction is horizontal. Thus, it has small demagnetization field. The front view of a horizontally magnetized ferrite CPW TL is shown in Figure 2. As shown, an external DC magnetic field is applied to the ferrite substrate in the: direction shown in the figure, inducing an internal magnetic field $\left(\mathrm{H}_{0}\right)$ which causes the ferrite substrate to have the saturation magnetization $\left(\mathrm{M}_{0}\right)$ in the same direction. For the shown direction of DC magnetic bias, the ferrite has a permeability tensor, $[\mu]$, given as:

where:

$$
[\mu]=\mu_{\mathrm{O}}\left[\begin{array}{ccc}
\mu & 0 & -j k \\
0 & 1 & 0 \\
j k & 0 & \mu
\end{array}\right]
$$

$$
\mu=\frac{\omega_{h m}^{2}-\omega^{2}}{\omega_{h}^{2}-\omega^{2}}, k=\frac{\omega \omega_{m}}{\omega_{h}^{2}-\omega^{2}}, \omega_{h}=\mu_{0} \gamma H_{0}, \omega_{m}=\mu_{0} \gamma M_{0}, \omega_{h m}=\mu_{0} \gamma \sqrt{H_{0}\left(H_{0}+M_{0}\right)}, \gamma
$$

is the gyromagnetic ratio of the ferrite and $\mu_{0}$ is the free space permeability. In this case, the effective relative ferrite permeability for horizontal magnetic bias can be expressed as:

$$
\mu_{f}=\mu
$$

It is shown that the effective permeability has a dispersion nature whose value is negative or positive depending on the applied magnetic field, the ferrite substrate specification and the DC magnetic bias.

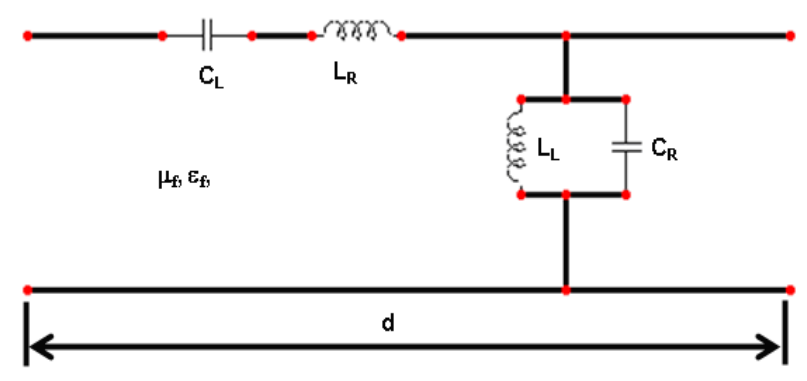

Fig. 1: The equivalent circuit model of a ferrite

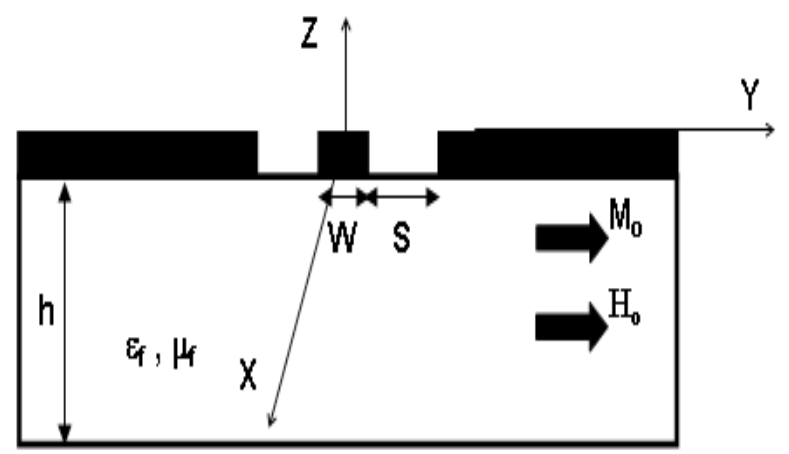

Fig. 2: Front view of the CPW TL on ferrite substrate horizontally magnetized

\section{TUNABLE COUPLED LINE COUPLER}

The backward coupling of a symmetrical CRLH coupled line coupler (CLC) has been explained using coupled line approach ${ }^{[30]}$ as: 


$$
C=\frac{A\left(\beta_{I}-\beta_{L H}\right) e^{-j \beta_{I} l}+B\left(\beta_{I I}-\beta_{L H}\right) e^{-j \beta_{I I} l}}{A\left(\beta_{I}-\beta_{L H}\right)+B\left(\beta_{I I}-\beta_{L H}\right)}
$$

where $\mathrm{A}$ and $\mathrm{B}$ are constants, $\beta_{\mathrm{I}, \mathrm{II}}$ are the coupled propagation constants defined in terms of the propagation constant along the hosting CRLH TL $\left(\beta_{\mathrm{LH}}\right)$ and the backward coupling coefficient between the two hosting coupled lines $\left(\mathrm{C}_{\mathrm{BW}}\right)$ as:

$$
\beta_{I, I I}=\sqrt{\beta_{L H}^{2}-C_{B W}^{2}}
$$

Since, as seen from (5), $\beta_{\mathrm{I}, \mathrm{II}}$ may be imaginary at some frequencies, hence, from (4), it can be seen that even 0 $\mathrm{dB}$ backward coupling is possible within these frequency bands. Thus, CRLH CLC can provide much higher coupling, up to $0 \mathrm{~dB}$, with relatively wide line separation over a broad bandwidth and very compact size, especially at lower frequencies compared to a conventional one $\mathrm{e}^{[2,3]}$. From ferrite CRLH TL concepts explained above, it can be claimed that, a ferrite CRLH CLC has dispersive $\left(\beta_{\mathrm{LH}}\right)$ and $\left(\mathrm{C}_{\mathrm{BW}}\right)$ due to the dispersive nature of the ferrite permeability. Consequently, a ferrite CRLH CLC has a dispersive backward coupling (C) as it can be seen from (4). Detailed explanation about this coupling mechanism is explained in ${ }^{[20]}$.

In our paper, we present a novel dual mode, tunable, and nonreciprocal ferrite CRLH backward CPW CLC. The proposed coupler was designed using two identical CRLH TLs over ferrite substrate as shown in Figure 3.An internal horizontal DC magnetic field $\left(\mathrm{H}_{0}\right)$ is applied to the ferrite substrate. The coupler's through output and the backward coupling output are located in two separated bands. The first mode was designed to demonstrate a reciprocal backward coupling propagation with almost $0 \mathrm{~dB}$ coupling level in the first band. The second band has nonreciprocal through propagation. Moreover, the coupler can be tuned by changing $\left(\mathrm{H}_{0}\right)$. The coupler was designed through comparing its equivalent circuit model simulating results to the real structure simulation results obtained using the commercial electromagnetic full wave simulation software ANSOFT- HFSS. The simulated scattering parameters of both the circuit model and the real layout structure using HFSS; for $\mathrm{H}_{0}=50,000 \mathrm{Oe}$, are shown in Figure 4. This very high DC magnetic bias is selected such that the ferrite is isotropic within the frequency band of interest; so that the equivalent circuit model is valid. The figure shows good agreement between the two simulated results, especially for the backward coupling level. The backward coupling level is approximately $-1 \mathrm{~dB}$, within the frequency band from 5 $\mathrm{GHz}$ to $9 \mathrm{GHz}$ (57\%). Considering the HFSS results, we can conclude within that frequency bandwidth, the coupler has approximately better than $-10 \mathrm{~dB}$ reflection coefficient, through propagation level below $-12 \mathrm{~dB}$, and better than 20 $\mathrm{dB}$ forward coupling isolation difference

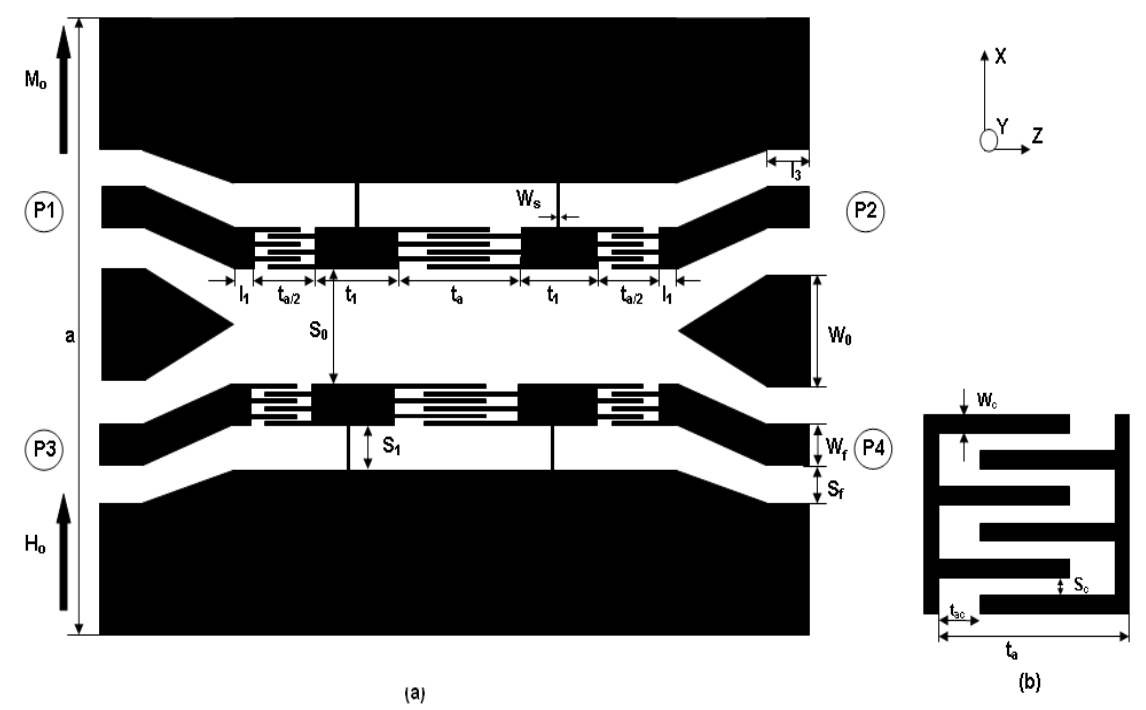

Fig. 3: The ferrite CRLH CPW CLC layout, $\mathrm{W}_{0}=6 \mathrm{~mm}, \mathrm{t}_{\mathrm{a}}=2 . \mathrm{mm}, \mathrm{t}_{1}=1.5 \mathrm{~mm}, \mathrm{~S}_{\mathrm{o}}=0.5 \mathrm{~mm}, \mathrm{~W}_{\mathrm{f}}=1.3 \mathrm{~mm}, \mathrm{~W}_{\mathrm{s}}=0.25 \mathrm{~mm}(\mathrm{~b})$. The interdigital capacitor geometry $\mathrm{S}_{\mathrm{c}}=\mathrm{Wc}=0.1 \mathrm{~mm}, \mathrm{t}_{\mathrm{ac}}=0.2 \mathrm{~mm}$. 


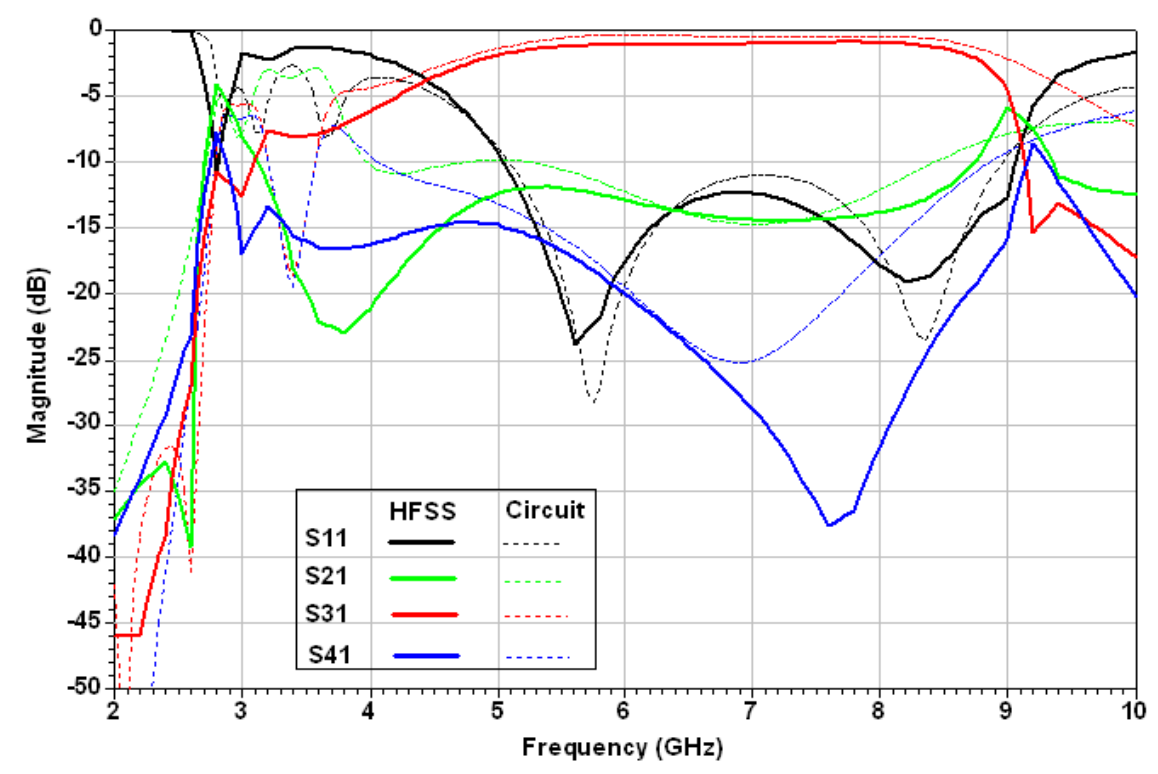

Fig. 4: HFSS (for $\mathrm{H}_{0}=50,000 \mathrm{Oe}$ ) and equivalent circuit simulated scattering parameter magnitudes of the ferrite CRLH CPW CLC.

By applying lower DC magnetic bias values, the upper cut off frequency of the backward coupling propagation will coincide with the onset of the negative permeability of the hosting CPW ferrite TL. Thus, the coupler can be tunable by changing $\mathrm{H}_{0}$. This can be confirmed by studying the CLC performance for different values of $\mathrm{H}_{0}$. For $\mathrm{H} 0=1750$ Oe, the results in Figure 5 (a), illustrates a backward propagation with a very close to $0 \mathrm{~dB}$ level over a frequency bandwidth that extends from $3.4 \mathrm{GHz}$ to $5.5 \mathrm{GHz}$. Then, it decreases from approximately $-3 \mathrm{~dB}$ at $5.7 \mathrm{GHz}$ with a very high slope to reach approximately -10 $\mathrm{dB}$ at $6 \mathrm{GHz}$. Following the backward propagation passband, the coupler has through propagation, no stopband separates them. On contract to the backward coupling performance, the through level increases from approximately $-13 \mathrm{~dB}$ at 6

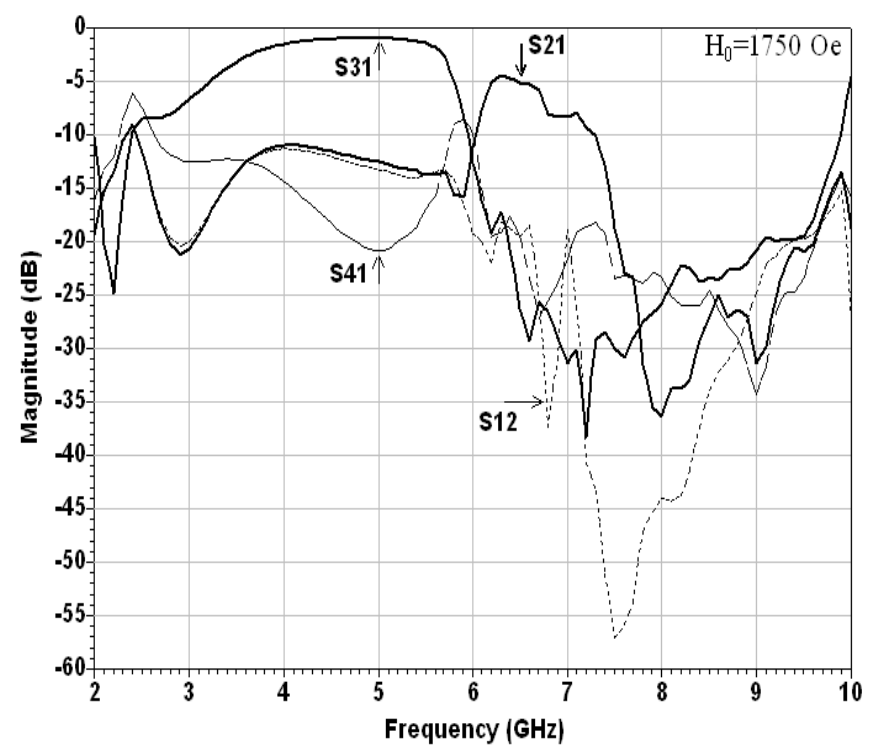

(a)
$\mathrm{GHz}$ up to $-5 \mathrm{~dB}$ at $6.2 \mathrm{GHz}$ with nonreciprocal propagation with nonreciprocity isolation level that extend up to 35 $\mathrm{dB}$ at $6.8 \mathrm{GHz}$. However, the through propagation level is some how lossy compared to the backward coupling in the first band due to the lossy nature of the ferrite substrate within the negative permeability frequency band. Finally, the coupler has low forward coupling signal (S41) whose isolation level is better than $20 \mathrm{~dB}$ over most of the operating bandwidths.

By changing $\mathrm{H} 0$ to $2250 \mathrm{Oe}$, As illustrated in Figure 5 (b), the $3 \mathrm{~dB}$ upper cut off frequency of the backward coupler propagation is increased to approximately 6.5 $\mathrm{GHz}$ while the nonreciprocal through propagation exists within the frequency band from $6 \mathrm{GHz}$ to approximately $8 \mathrm{GHz}$.

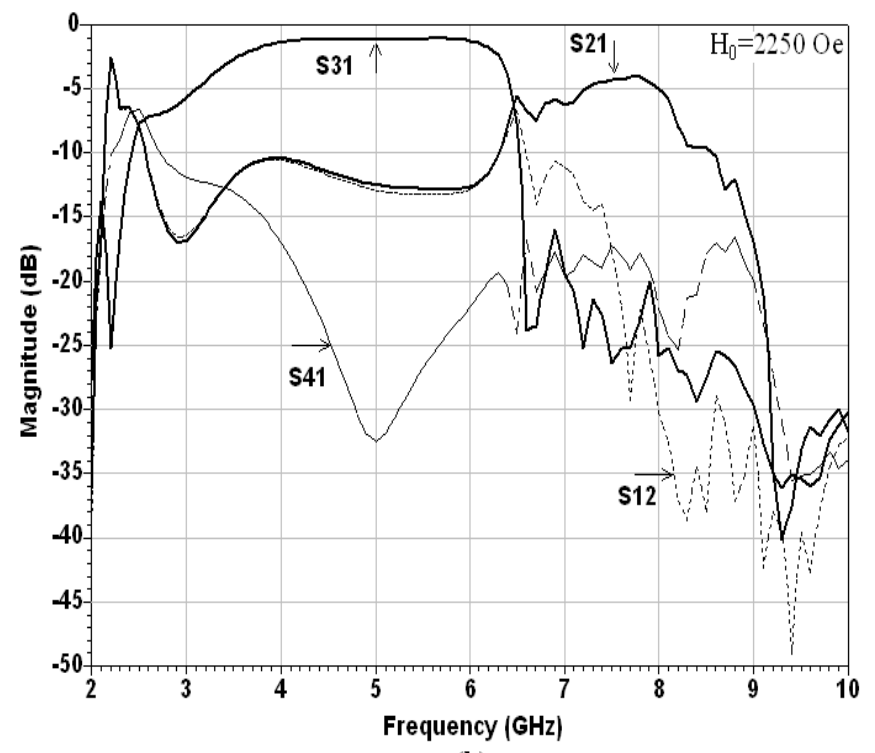

(b)

Fig. 5: The full wave simulated scattering parameter magnitudes of the dual mode ferrite CRLH CPW CLC for (a) $\mathrm{H}_{0}=1750$ Oe and (b) $2250 \mathrm{Oe}$ 


\section{TUNABLE WIDE BAND IMPEDANCE TRANSFORMER}

A compact quarter wavelength CRLH transformer can be designed using a CRLH TL. The CRLH transformer can satisfy arbitrary $\pm 900^{\circ}$ phase shift controlled by the CRLH elements. The dispersive ferrite permeability can be utilized to design a wide band tunable ferrite CRLH transformer. The upper cut off frequency of the transformer was setting by the selection of the applied DC magnetic bias value to be the onset of the lossy propagation within the negative ferrite permeability frequency band. The transformer consists of only a CRLH unit cell formed by series air gap capacitors and a shunt meandered line inductor whose detailed sketch is shown in Figure 6. The simulated scattering parameters of the proposed CRLH transformer is for $\mathrm{H}_{0}=2000$ Oe,
2250 Oe, and 2500 Oe as shown in Figure 7. It is clear that the both of the lower cut off and operating centre frequencies of the transformer are increasing by increasing H0. On the other hand, the upper cut off frequency is kept constant at $7 \mathrm{GHz}$. Within the operating bandwidth, the insertion loss is close to $0 \mathrm{~dB}$ at its perfect matching, centre, frequency while it increases to no more than $1.5 \mathrm{~dB}$ over the rest bandwidth. On the other hand, return loss is close to $20 \mathrm{~dB}$ at the perfect matching frequencies while its worst case value, over the whole operating bandwidth is $8 \mathrm{~dB}$, at which the insertion loss is $1.2 \mathrm{~dB}$ which still satisfy a reasonable transformer operation condition. The results confirm that the transformer can match a $25 \Omega$ load and a $50 \Omega$ line with tunable nature overa wide bandwidth (more than $64 \%$ ) by changing the applied DC magnetic bias.

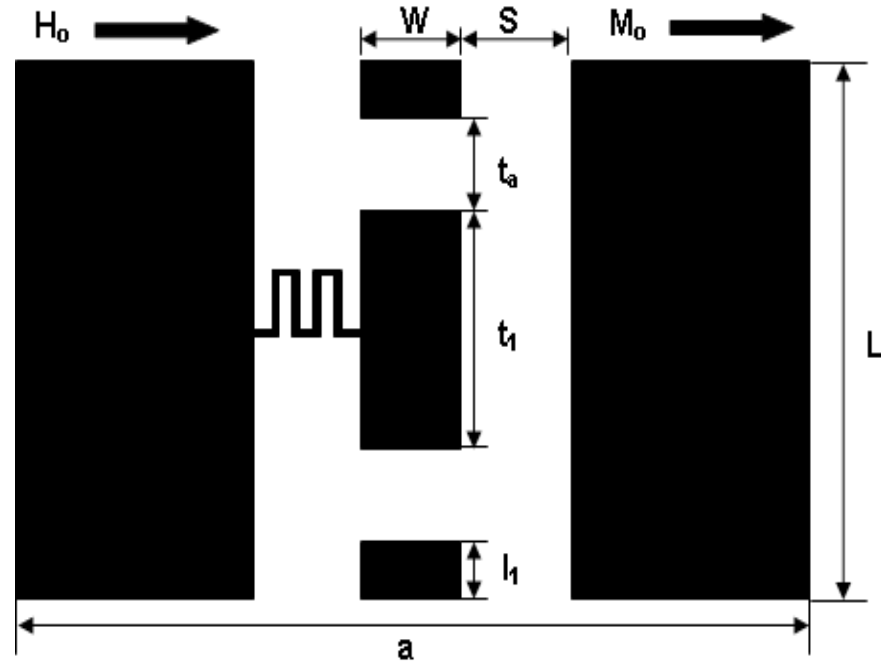

(a)
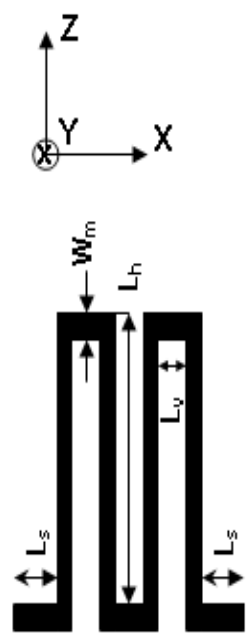

(b)

Fig. 6: (a) The layout geometry of the ferrite CRLH CPW transformer $\mathrm{a}=30 \mathrm{~mm}, \mathrm{~L}=1.7 \mathrm{~mm}, \mathrm{~W}=1.8 \mathrm{~mm}, 1_{1}=0.5 \mathrm{~mm}, \mathrm{t}_{\mathrm{a}}=0.1 \mathrm{~mm}, \mathrm{t}_{1}=0.5 \mathrm{~mm}$. (b) The meandered line inductor geometry.

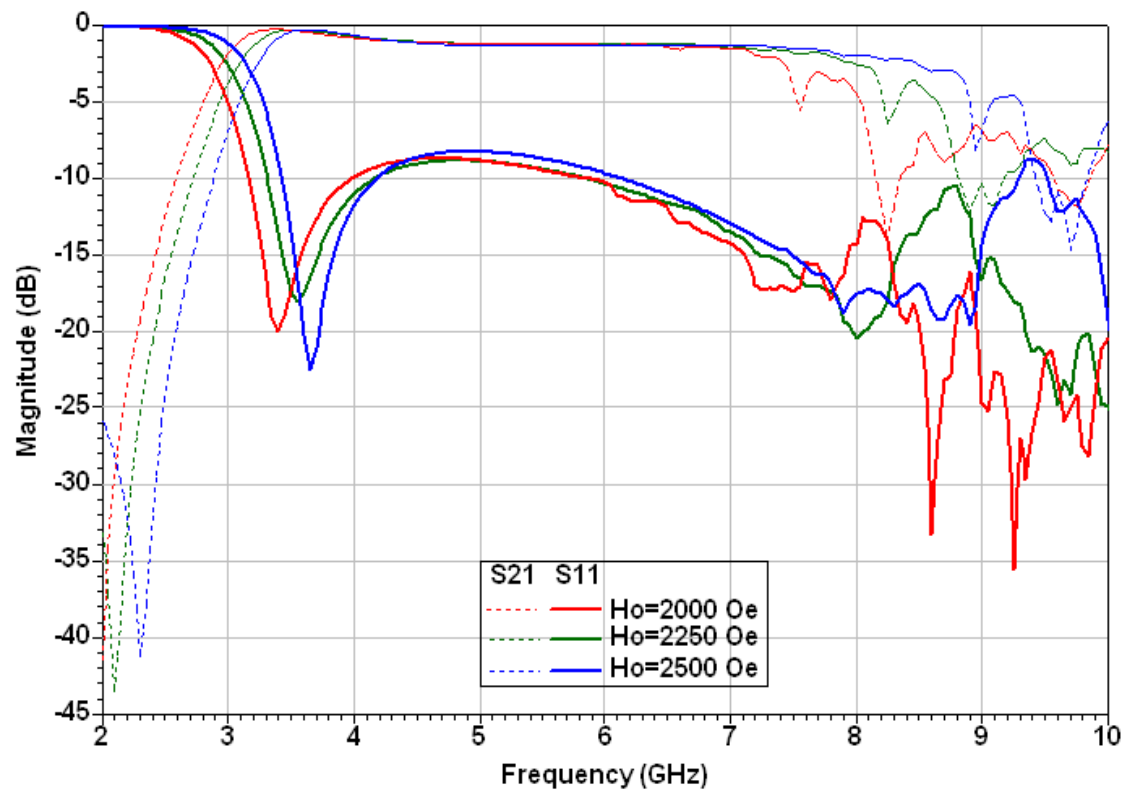

Fig. 7: The full wave scattering parameters magnitudes of the wide band ferrite transformer for different $\mathrm{H}_{0}$ values. 


\section{TUNABLE Microwave Resonator}

A ferrite CRLH CPW zeroth order resonator can be designed to be tunable by varying the applied DC magnetic bias. The layout of the ferrite CRLH CPW zeroth order resonator is shown in Fig. 1. The zeroth order resonator is then connected to a $2 \mathrm{~mm}$ long $50 \Omega \mathrm{CPW}$ TL through a small coupling air gap capacitor. The resonator length is $5.25 \mathrm{~mm}$ which is only $0.175 \lambda \mathrm{g}$, the length of the
CRLH resonator including the air gap capacitor is 6.25 $\mathrm{mm}(0.2 \lambda \mathrm{g})$, calculated at $4 \mathrm{GHz}$. Compared to half wavelength conventional resonator, the CRLH zeroth order resonator is only $35 \%$ in size. An internal DC magnetic field $\left(\mathrm{H}_{0}\right)$ is applied to the ferrite substrate in the direction shown in Fig. 8, resulting in the ferrite substrate to have the saturation magnetization in the same direction

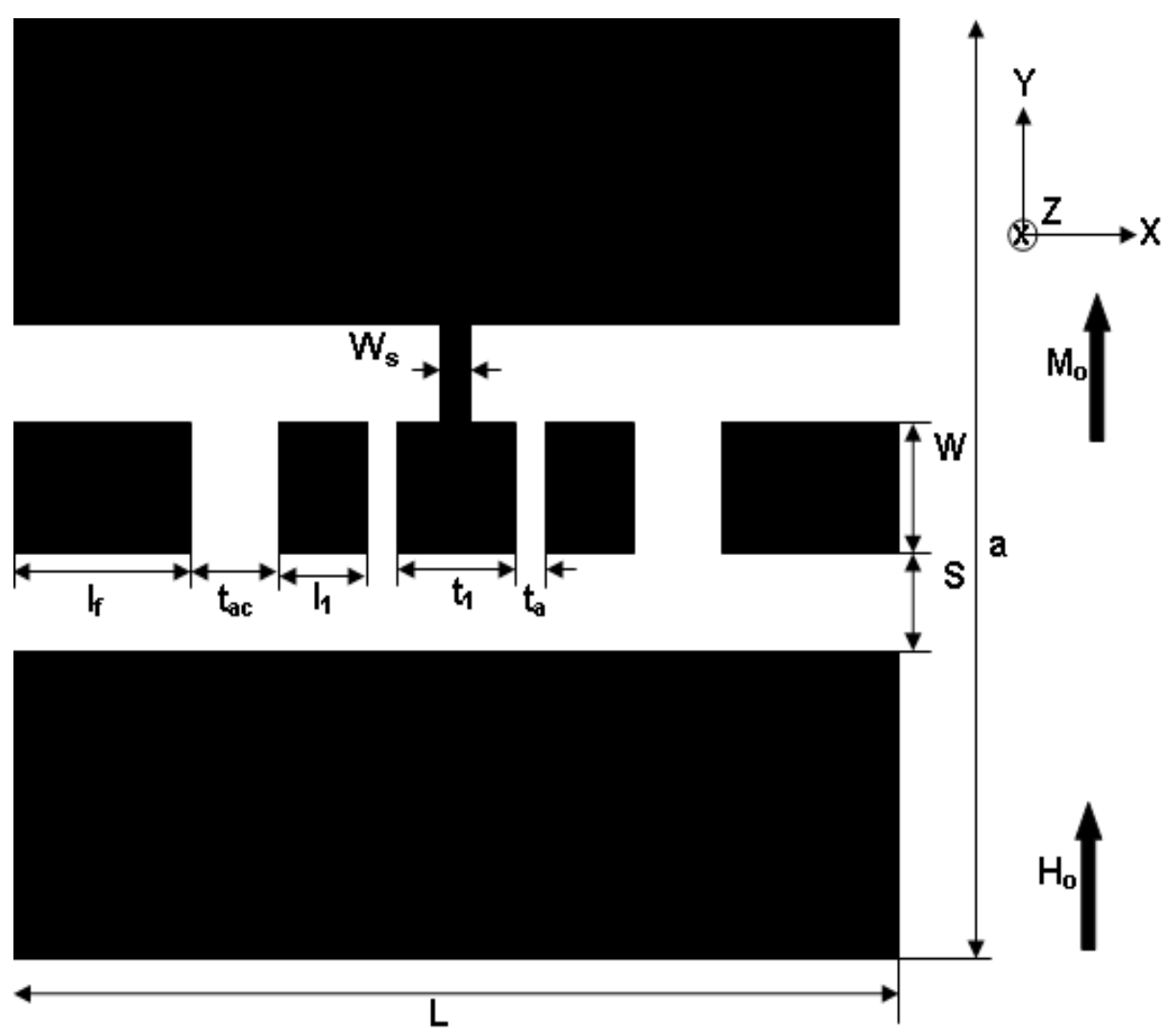

Fig. 8: The layout of the ferrite CRLH CPW zeroth order resonator $\mathrm{a}=19.8 \mathrm{~mm}, \mathrm{~L}=10.25 \mathrm{~mm}, \mathrm{~W}=2 \mathrm{~mm}, \mathrm{~S}=1 \mathrm{~mm}, \mathrm{t}_{1}=1 \mathrm{~mm}, \mathrm{ta}=0.125 \mathrm{~mm}$, $\mathrm{t}_{\mathrm{ac}}=0.5 \mathrm{~mm}, \mathrm{l}_{1}=2 \mathrm{~mm}, \mathrm{l}_{\mathrm{f}}=2 \mathrm{~mm}, \mathrm{~W}_{\mathrm{s}}=0.25 \mathrm{~mm}$.

The principle of its operation is based on cascading a CRLH TL section and a RH one. The total phase shift of the TL can be expressed as the sum of CRLH and RH phase shifts, i.e.

$$
\beta l=\beta_{l} d_{l}+\beta_{r} d_{r}
$$

Where $\beta_{l}$ and $\beta_{r}$ are the propagation constants of CRLH and RH sections, respectively, whereas $d_{1}$ and $d_{\mathrm{r}}$ represent the lengths of these sections, respectively, while $l$ is the total length of the combined line. The resonant condition of the resonator shown in Fig. 2 can be found by setting "(1)," equal to $n \pi$ i.e.,

$$
\beta l=\beta_{l} d_{l}+\beta_{r} d_{r}=n \pi
$$

To investigate the tuning capability of the CRLH CPW ZOR by changing the DC magnetic bias, the ZOR was simulated for more varying DC magnetic bias in the range between 1000 Oe to 5000 Oe. The simulated scattering parameters in these cases are shown in Fig. 9. The figure shows that the resonant frequency changes by changing the DC magnetic bias. It can be seen the insertion loss characterizing the proposed resonator is better than $3 \mathrm{~dB}$ except for the two lower $\mathrm{H} 0$ cases of $1000 \mathrm{Oe}$ and $1125 \mathrm{Oe}$ at which the insertion loss is $13 \mathrm{~dB}$ and $5 \mathrm{~dB}$, respectively. The reason for that is the overlap between the resonant frequency and the high lossy negative permeability of the hosting ferrite TL at their resonant frequency which is clearer for $\mathrm{H}_{0}=1000$ Oe.

Also, the proposed ferrite CRLH CPW ZOR is demonstrating a better than $10 \mathrm{~dB}$ return loss at the resonant frequency, except for the two aforementioned lowered cases where it is only $3 \mathrm{~dB}$ and $7 \mathrm{~dB}$ for $\mathrm{H}_{0}=1000$ Oe and 1125 Oe, respectively. 


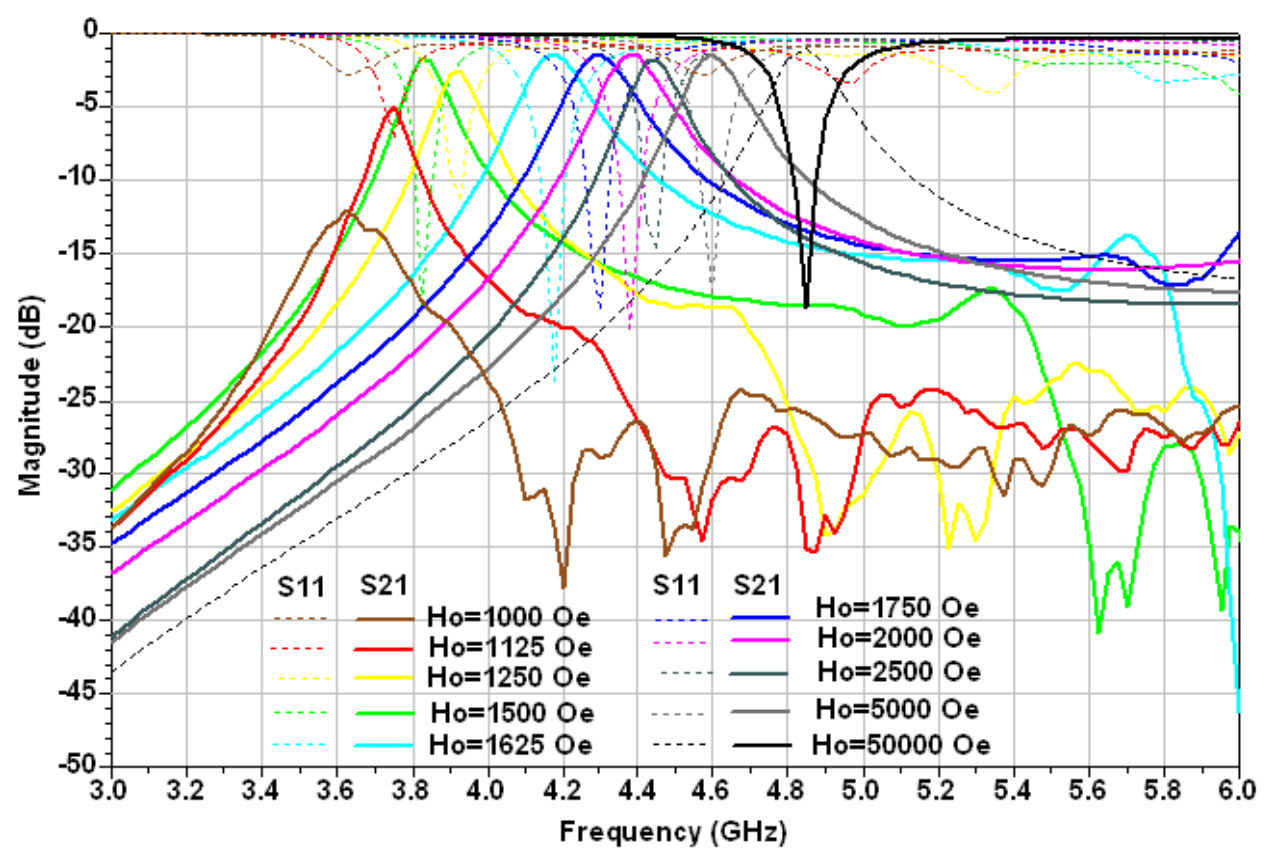

Fig. 9: The full wave scattering parameter magnitudes of the ferrite CRLH CPW ZOR at different DC magnetic bias.

As an examples for an applying the DC magnetic bias of $1250 \mathrm{Oe}$, the ZOR resonates at a resonant frequency of 3.9 $\mathrm{GHz}$. At this resonant frequency, the resonator has a return loss close to $15 \mathrm{~dB}$ and an insertion loss better than $3 \mathrm{~dB}$. In another case for the applying the DC magnetic bias of 2000 $\mathrm{Oe}$, the resonant frequency has been shifted to $4.4 \mathrm{GHz}$ with return loss better than $20 \mathrm{~dB}$ and insertion loss also better than $3 \mathrm{~dB}$. It is evident that the resonant frequency can be tuned by changing the applied DC magnetic bias. Similar results can be observed in the figure for the other DC magnetic bias values.

The variation of the resonant frequency against the applied DC magnetic field bias is made clear in Fig. 10. It can be seen that the resonant frequency exhibits a nonlinear variation with the applied DC magnetic bias. The resonant frequency increases with increasing the DC magnetic bias from $3.6 \mathrm{GHz}$ at $\mathrm{H}_{0}=1000$ Oe to $3.95 \mathrm{GHz}$ at $\mathrm{H}_{0}=1250 \mathrm{Oe}$, then it decreases to $3.85 \mathrm{GHz}$ at $\mathrm{H}_{0}=1500 \mathrm{Oe}$. Beyond this point the resonant frequency starts to increase again with increasing H0 until the DC bias reaches its typical value where the ferrite is characterized through its isotropic electrical properties, corresponding to the resonant frequency of $4.8 \mathrm{GHz}$.

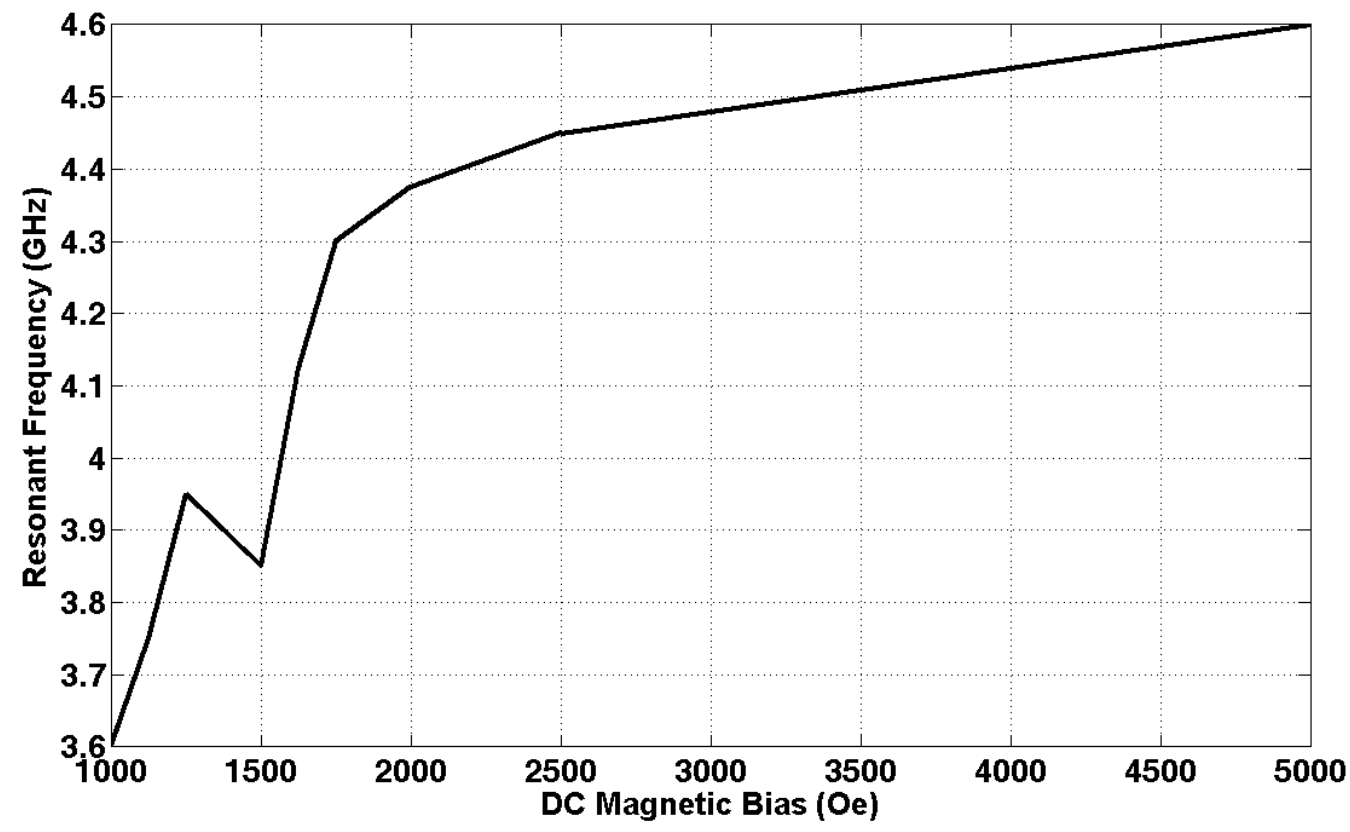

Fig. 10: The resonant frequency variation against the DC magnetic bias. 


\section{CONCLUSION}

The paper introduces examples of novel microwave CRLH devices. These devices combine the novel CRLH properties and the ferrite properties to introduce novel microwave components demonstrating compact size, broadband and novel magnetic properties. First, the basics of ferrite CRLH TL are reviewed. A tunable and nonreciprocal ferrite CRLH CPW CLC is introduced. The CLC is compact in size and has broad band (up to 57\%). Also, a compact and wideband CRLH tunable transformer (more than 64\%) is introduced. Finally, an ultra compact $(0.2 \lambda \mathrm{g})$ zeroth order resonator with tunable properties is presented.

\section{REFERENCES}

[1] Veselago V. G., "The electrodynamics of substances with simultaneously negative values of $\mathcal{E}$ and $\mu$," Soviet Physics - Uspekhi Vol.10, No.4, 509-514, 1968

[2] Smith D. R., W. J. Padilla, D. C. Vier, S. C. Nemat-Nasser and S. Schultz, "Composite medium with simultaneously negative permeability and permittivity," Phys. Rev. Lett., Vol.84, No.18, 4184-4187, 2000.

[3] Caloz C., T. Itoh, Electromagnetic Metamaterials Transmission Line Theory and Microwave Applications. New Jersey: John Wiley and Sons, 2006.

[4] Eleftheriades G. V., K. H. Balmin, Negative Refractive Metamaterials. New Jersey: John Wiley and Sons, 2005.

[5] Tsutsumi M. and T. Ueda, "Nonreciprocal left-handed microstrip lines using ferrite substrate," in Proceedings of IEEE MTT-S Int. Microwave Symposium, Fort Worth, USA, June 2004, 249-252.

[6] Abdalla M. and Z. Hu, "On the study of CWP dual band left handed propagation with reciprocal and nonreciprocal characteristics over ferrite substrates," in Proceedings of IEEE AP-S Int. Symp, Honolulu, USA, June 2007, 2578-2581.

[7] M. Tsutsumi and T. Ueda "Nonreciprocal left-handed microstrip lines using ferrite substrate," in Proc IEEE MTT-S Int. Symp., USA, 2004, pp. 249-253.

[8] M. Tsutsumi and T. Ueda "Left handed transmission characteristics of ferrite microstrip lines without series capacitive load," IEICE Trans. Electron., vol. E89, pp. 1318-1323, Sep. 2006.

[9] M. Abdalla, and Z. Hu, "On the study of CWP dual band left handed propagation with reciprocal and nonreciprocal characteristics over ferrite substrates," in IEEE AP-S Int. Symp., USA, 2007, pp. 2578-2581.

[10] G. Sajin, I. A. Mocanu, F. Craciunoiu, M. Carp," MM-wave lefthanded transmission line antenna on anisotropic substrate" 43rd European Microwave Conference (EuMC), 2013, pp. 668-671.

[11] ${ }^{\varepsilon e}$ Y.-J Huang, G. Wen, T. Q. Li, Li, J.L. Kang Xie, "Design and Characterization of Tunable Terahertz Metamaterials With Broad Bandwidth and Low Loss", IEEE Antennas and Wireless Propagation Letters, vol. 11, pp. 264-267, 2012.

[12] B. Lax, and K. J. Button, Microwave Ferrites and Ferrimagnetics, New York: McGraw Hill, 1962.
[13] M. A. Abdalla and Z. Hu, "Multi-band functional tunable LH impedance transformer," Journal of Electromagnetic Wave and Applications, vol. 23, pp. 39-47, 2009.

[14] M. A. Abdalla and Z. Hu, "Compact tunable left handed ferrite transformer," International Journal of Infrared and Millimeter Waves, vol.30, no. 8, pp.813-825, 2009 .

[15] M. Abdalla and Z. Hu, " Compact Novel CPW Ferrite Coupled Line Circulator with Left-handed Power Divider/Combiner ", 2011 European Microwave Week, EuMW, Digest, UK, pp. 794-797.

[16] T. Kodera, and C. Caloz, "Integrated Leaky-Wave Antenna-Duplexer/ Diplexer Using CRLH Uniform Ferrite-Loaded Open Waveguide", IEEE Trans. Antenna and Propagation vol. 58, no. 8, pp. 2508-2514, 2010.

[17] T. Kodera, D. L. Sounas, D.L. and C. Caloz, "Tunable magnetless non-reciprocal metamaterial (MNM) and its application to an isolator" 2012 Asia-Pacific Microwave Conference Proceedings (APMC), pp. 73-75, 2012.

[18] M. Abdalla and Z. Hu "Ferrite Tunable Metamaterial Phase Shifter", 2010 IEEE AP-S International Antenna and Propagation Symposium Digest, Jul. 11-17, 2010, Toronto, Canada, pp. 1-4.

[19] M. Tsutsumi, K. Okubo, "Effect of stubs on ferrite microstrip line magnetized to wave propagation", APMC 2009. Asia Pacific Microwave Conference, 2009, pp. 1234-1237

[20] M. A. Abdalla and Z. Hu, "Compact tunable single and dual mode ferrite left-handed coplanar waveguide coupled line couplers, "IET Microwaves, Antennas and Propagation -June vol. 3, no. 4, p. 695-702, 2009.

[21] M. Abdalla and Z. Hu, "Composite Right/Left-handed Coplanar Waveguide Ferrite Forward Coupled-Line Coupler, "IET Microwaves, Ant. and Propagation, vol 9, no. 10, pp. 1104-1111, 2015.

[22] S. Karimian, M. Abdalla and Z. Hu, "Tunable Metamaterial Ferrite Stepped Impedance Resonator (SIR)," 27th Progress in Electromagnetics Research Symposium, 2010, Xi'an, China, pp 165-168.

[23] M. A. Abdalla and Z. Hu, "Compact Metamaterial Coplanar Waveguide Ferrite Tunable Resonator,"IET Microwaves, Antennas \& Propagation, vol. 10, no. 4, pp. 406-412, 2016.

[24] M. Abdalla, and Z. Hu, "Design and Analysis of Tunable Left Handed Zeroth Order Resonator on Ferrite Substrate," IEEE Trans. Magnetics. vol 44, pp. 3095-3098, Nov. 2008.

[25] J. Ghalibafan and Nader Komjani, "Tunable zeroth-order resonator based on a ferrite metamaterial structure", Chinese Physics B, vol. 22, no. 10, pp. 107805-1 - 107805-5, 2013.

[26] A. Zermane, B. Sauviac, B. Bayard, B. P. Gervy, J. J. Rousseau and A. Benghalia, "Experimental verification of tunable property of a zerothorder resonator on ferrite substrate", Microwave and Optical Technology Letters, vol. 56, no. 12, pp. 2805-2809, 2014.

[27] M. Abdalla and Z. Hu, "Compact and Tunable Metamaterial Antenna for Multi-band Wireless Communication applications", 2011 IEEE AP-S Int. Ant. and Prop. Symp. USA, pp. 2951-2953.

[28] A. Porokhnyuk, T. Ueda, Y. Kado, Y.and T. Itoh, "Design of nonreciprocal CRLH metamaterial for non-squinting leaky-wave antenna" 2013 IEEE MTT-S Int. Microwave Symposium IM-S, pp. 1-3.

[29] Mahmoud Abdelrahman Abdalla. "Ferrite left-handed meta-materials for RF microwave applications." PhD diss., Manchester University, 2008. [30] Nguyen H. V. and C. Caloz, "Generalized coupled-mode approach of metamaterial coupled-line couplers: coupling theory, phenomenological explanation, and experimental demonstration," IEEE Transactions on Microwave Theory and Techniques, vol. 55 , pp. 1029-39, 2007 